\section{Carotid artery disease presenting as cough headache in a patient on haemodialysis}

\section{Sir,}

Headache has been associated with all types of cerebrovascular events, such as transient ischaemic attacks, lacunar infarcts and parenchymal haemorrhage.' In addition, the association of headache to carotid artery disease is well recognized, although the mechanisms of pain remain unclear. ${ }^{2}$ Headache has been reported after spontaneous carotid dissection and carotid endarterectomy. ${ }^{4}$

Cough headache is a well described entity consisting of transient pain on coughing, sneezing, laughing or heavy lifting. This is usually a benign idiopathic state although it is occasionally associated with foramen magnum and posterior fossa pathology (Arnold Chiari, arteriovenous malformations and tumour). ${ }^{1}$

To our knowledge, there is only one previous report concerning cough headache and carotid artery disease. ${ }^{5}$ We report a patient on haemodialysis with cough headache as the presenting symptom of carotid artery disease.

A 54 year old patient had been on haemodialysis (HD) for 10 years because of chronic renal failure (CRF) secondary to membranous nephropathy. He had a history of heavy smoking, chronic obstructive lung disease and long-term hypertension. Clinical features of generalized atherosclerosis developed in 1987, consisting of angina pectoris and intermittent claudication.

In July 1988 he began to suffer from right severe retrocular pain following cough attacks. In November, right blunted vision, weakness of the left arm with abnormal spontaneous movements of his left hand, minimal facial paresis and dysarthria were associated with cough headache. Those symptoms appeared not only with cough, but also with intradialysis hypotension (systolic blood pressure lower than $80 \mathrm{mmHg}$ ). Neurological symptoms reversed completely in 10-15 minutes. He was admitted to hospital in December. Physical examination revealed a chronically ill patient. Bilateral carotid bruits were heard. Peripheral pulses were absent. Neurological examination was unremarkable. Computerized tomography of the head excluded intracranial pathology such as tumour, posterior fossa disease and hydrocephalia. The electroencephalogram was normal. Carotid Doppler showed significant stenosis of left common carotid artery. Carotid arteriogram was proposed but the patient refused. Treatment with oral platelet antiaggregants and codeine was instituted, plus manoeuvres to avoid intradialysis hypotension, with transient improvement. In January 1989 he died following a myocardial infarction.

Our patient, like the one reported by Britton and Guiloff,' had had cough headache for several months before other neurological symptoms developed. The relation of the cough headache to the carotid artery stenosis is uncertain. In our case, headache and neurological symptoms always followed cough and hypotension. These are situations that decrease cerebral flow and may cause cerebral ischaemic events in the presence of a severely stenosed carotid artery.

In conclusion, cough headache can be the presenting symptom of carotid obstruction even before focal symptoms develop. We believe that in patients with cough headache, carotid arteriogram must be considered in order to exclude carotid pathology.

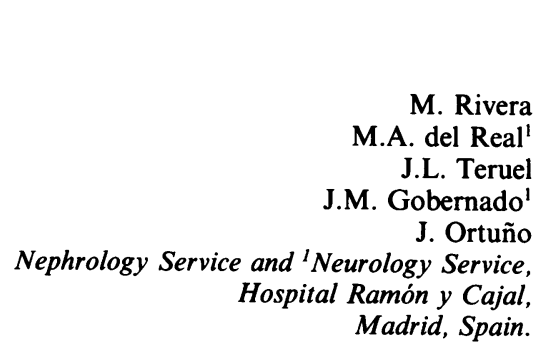

\section{References}

1. Ekbom, K. Cough headache. In: Rose, C. (ed) Handbook of Clinical Neurology, Vol. 4 (48). Headache. 1986, pp. 367-371.

2. West, T.E.T., Davies, R.J. \& Kelly, R.E. Horner's syndrome and headache due to carotid artery disease. Br Med J 1976, i: 818-820.

3. Messert, B. \& Black, J.A. Cluster headache, hemicrania and other head pains: Morbidity or carotid endarterectomy. Stroke 1978, 9: 559-562.

4. Edmeads, J. The headaches of ischemic cerebrovascular disease. Headache 1979, 19: 345-349.

5. Britton, T.C. \& Guiloff, R.J. Carotid artery disease presenting as cough headache. Lancet 1988, i: 1406-1407.

\section{Hypereosinophilia and acute lymphocytic leukaemia}

Sir,

The association of acute lymphocytic leukaemia (ALL) and eosinophilia is a rare event. Few cases characterizedo phenotypically and genotypically have been reported. ${ }^{1,2}$ We observed a hypereosinophilic syndrome as the firstoㅡㄴ finding in a patient who developed an ALL of pre-B lineage.

A previously healthy 15 year old male was admitted to our hospital because of chest pain, fever and diarrhoea of acute onset. Physical examination was normal; no cutaneous lesions, enlargement of lymph nodes, spleen or liver were noted. The peripheral blood counts showed a haemoglobin of $12.8 \mathrm{~g} / \mathrm{dl}$, platelets of $121 \times 10^{9} / 1$ and WBC of $95.7 \times 10^{9} / 1$ with $87 \%$ eosinophils $\left(83 \times 10^{9} / 1\right)$, many of them were abnormal with vacuoles and partially degranulated; no immature cells were observed in the blood smear, serum lactate dehydrogenase was $469 \mathrm{U} / 1$. Haemostatic parameters, electrocardiogram and chest $\mathrm{X}$-ray were normal. Bone marrow aspirate showed $30 \%$ lymphoid blasts and $40 \%$ eosinophils, some of them abnormal. The blastic population was myeloperoxidase, chloro-acetate-esterase, butyrate-esterase and cyanideresistant myeloperoxidase negative in the cytochemical stains. The CD10 and TdT, determined by immunoperoxidase technique, ${ }^{3}$ were positive. Other surface markers analysed by flow cytometry showed the HLA-DR positive in $86 \%, C D 19$ in $86 \%$, and CD20 in $79 \%$ of the bone marrow mononucleated cells; CD3, CD2 and CD14 were positive only in $5 \%, 6 \%$ and $3 \%$ of the cells. Bone marrow chromosomal analysis (G-banding) showed a normal karyotype ( $46 \mathrm{XY})$ in 20 studied metaphases. A diagnosis of common/preB-ALL was made. The patient achieved complete remission in 4 weeks with vincristine, daunorubicin, L-asparaginase and prednisone; peripheral and bone marrow eosinophils disappeared. He remains in complete remission under chemotherapy 6 months after diagnosis. 